Revista de Investigación Educativa 26

enero-junio, 2018 | ISSN 1870-5308 | Xalapa, Veracruz

Instituto de Investigaciones en Educación | Universidad Veracruzana

\title{
Experiencias estudiantiles en la estrategia didáctica de aprendizaje invertido
}

\section{Student experiences in the didactic strategy of flipped learning}

\author{
Elvia Garduño Teliz \\ Colette Marie Dugua Chatagner ${ }^{b}$
}

Recibido: 27 de octubre de 2016 Aceptado: 25 de septiembre de 2017

El objetivo de esta investigación es describir la aplicación del aprendizaje invertido como estrategia didáctica en estudiantes de Licenciatura en Ciencias de la Educación de la Universidad Autónoma de Guerrero. El aprendizaje invertido es un sistema de acciones centrado en los intereses de los estudiantes y su empoderamiento; reconoce su capacidad de tomar decisiones basadas en el compromiso de realizar acciones cognitivas simples y complejas a través de retos en espacios múltiples. Se aplicó la investigación proyectiva de carácter cualitativo basada en la observación participante de ambientes de aprendizaje mixtos en los que se realizaron actividades autogestivas y colaborativas. Los procesos reflexivos involucraron el análisis de contenido para identificar categorías en la planeación, implementación y evaluación de la estrategia. Los resultados revelan una corresponsabilidad formativa y a la vez contradictoria respecto a los roles y responsabilidades asumidos por los estudiantes; también identifican acciones de mejora para el aprendizaje invertido.

Palabras clave: Aprendizaje; estrategias didácticas; estudiantes.

\footnotetext{
a Candidata a Doctora en Pedagogía, Profesora Investigadora, Universidad Autónoma de Guerrero, México.凶 elvia_garduno_teliz@hotmail.com

b Doctora en Pedagogía, Profesora Investigadora, Universidad Autónoma de Guerrero, México. 凶dugua01@yahoo.com
} 
The aim of this research is to describe the application of flipped learning as a didactic strategy in undergraduate students in Education Sciences of the Autonomous University of Guerrero. Flipped learning is a system of actions focused on the interests of students and their empowerment by recognizing their ability to make decisions based on the commitment to perform simple and complex cognitive actions through challenges in multiple spaces. Qualitative projective research was applied, based on participant observation of mixed learning environments in which self - management and collaborative activities were carried out. Reflective processes involved content analysis to identify categories in strategy planning, implementation, and evaluation. The results reveal a formative and at the same time contradictory responsibility towards the roles and responsibilities assumed by the students, they also identify improvement actions for flipped learning.

Keywords: Learning; didactic strategies; students.

\title{
Experiencias estudiantiles en la estrategia didáctica de aprendizaje invertido
}

\author{
Student experiences in the didactic strategy of flipped learning
}

\section{Introducción}

$\mathrm{C}$ on la integración de la tecnología, la pedagogía y la didáctica a los espacios formativos, se han planteado actualizaciones y adaptaciones a los paradigmas educativos existentes. A partir de estos planteamientos se han generado diferentes perspectivas orientadas a diversificar de manera creativa e innovadora los procesos de enseñanza y aprendizaje. Uno de ellos es el aprendizaje invertido.

Esta propuesta, cuenta con diversas denominaciones; para algunos es un modelo (Nederveld \& Berge, 2015; Sales \& Pinto, 2016), porque conecta la teoría con la práctica en una forma homologada de realizar la práctica pedagógica y la didáctica; para 
otros es un enfoque pedagógico (Talbert, 2014) que aglutina diversas perspectivas de enseñanza y aprendizaje e introduce cambios a las mismas mediante las TIC. También se concibe como un método pedagógico (Bishop \& Verleger, 2013) porque emplea una serie de procedimientos que combinan técnicas y actividades didácticas.

Sea cual fuere su denominación, el aprendizaje invertido ha sido objeto de múltiples investigaciones en diferentes niveles educativos. Vidal, Rivera, Nolla, Morales y Vialart (2016) ubican al aula invertida como una estrategia didáctica que integra la instrucción directa con el aprendizaje constructivista, en la cual realizan una búsqueda de espacios virtuales como referentes. Aunque no hacen un señalamiento directo al aprendizaje invertido, establecen una relación entre la estrategia y la formación obtenida en diferentes niveles y espacios de aprendizaje. Por otro lado, Flores, Montaño, Herrera, Endoqui y García (2017) visualizan un modelo de gestión de aprendizaje invertido en el que realizan un estudio cualitativo (basado en la triangulación de la observación activa y la entrevista semiestructurada en niños y docentes de primaria para el logro de competencias comunicativas) en el cual manifiestan resultados favorables en la participación activa de ambos actores y en el desarrollo del pensamiento crítico. En las referencias consultadas, en estudiantes universitarios el aprendizaje invertido se han empleado a partir de estudios de caso orientados a la identificación de los estilos de aprendizaje, en cuyos resultados se reflejó un fuerte arraigo conductista por parte de los estudiantes (Santos, 2016).

Finalmente, desde la perspectiva de Sales y Pinto (2016) se presentan algunas preocupaciones sobre el aprendizaje invertido, tales como: a) la falta de tiempo en las habilidades de gestión; b) falta de acceso en tecnología apropiada; c) las dificultades de adaptación y la resistencia en los estudiantes. Todas ellas, son consideradas como parte del proceso de diseño, implementación y evaluación de la estrategia didáctica del aprendizaje invertido.

La investigación aborda la perspectiva de los estudiantes de la Licenciatura en Ciencias de la Educación en tanto futuros profesionistas en este ámbito; además de una mirada desde la didáctica en el proceso de diseño e implementación del aprendizaje invertido como estrategia, por lo que adquiere singular relevancia para ser replicada en otros contextos similares.

En el contexto de esta investigación se consideró pertinente emplear el término estrategia, puesto que se incluyen componentes cognitivos y operativos para el logro de una intención formativa. En este aspecto, la estrategia constituye un procedimiento o conjunto de pasos intencionados. De acuerdo con Díaz-Barriga y Hernández (2002), estos pasos "se encuentran involucrados en la promoción de aprendizajes sig- 
nificativos a partir de los contenidos escolares [...] y en las estrategias de aprendizaje, la responsabilidad recae en el aprendiz" (p. 80).

El término estrategia implica un "sistema de acciones para alcanzar los objetivos, tanto en lo personal, o grupal, como en la institución escolar" (Sierra, 2008, p. 28), es decir, involucra lo que se hace dentro y fuera del aula para aprender, con o sin la mediación docente.

Se realizó una investigación proyectiva de carácter participativo en la que los estudiantes llevaron a cabo una valoración experiencial, reflexiva y propositiva del proceso mediante la metodología cualitativa.

Aunque la metodología cualitativa es de carácter inductivo, el estudio presenta aspectos replicables para mejorar los procesos de enseñanza y aprendizaje, desde la experiencia de los futuros profesionales de la Escuela Superior de Ciencias de la Educación, quienes podrán ejercer, como parte de su carrera profesional: la docencia, el diseño curricular y la innovación en los procesos formativos a Nivel Medio Superior o Superior. En este contexto, el estudio aporta, desde la mirada de los estudiantes, orientaciones didácticas para aplicar el aprendizaje invertido tanto en ambientes virtuales como presenciales.

Otro aspecto de este trabajo es la diferenciación que se establece entre los conceptos de aprendizaje invertido y aula invertida. En una revisión del aprendizaje invertido o flipped learning publicada por la Universidad de George Mason en Estados Unidos, Yarbro, Arfstrom, McKnight y McKnight (2014) advierten sobre el riesgo de considerar al aula invertida y al aprendizaje invertido como sinónimos, puesto que una clase invertida puede no necesariamente conducir a un aprendizaje invertido, [ya que] muchos profesores pueden invertir sus clases al pedir a sus estudiantes leer textos, observar videos suplementarios o resolver problemas adicionales fuera de clase, quizá como tarea durante su estancia en la sala de estudio o en el autobús en el que se dirigen a un juego. (p. 5)

Sin embargo, sólo se plantea un cambio en la dinámica de la clase, es decir, una clase invertida, cuyo impacto será directamente proporcional a la realización de las tareas encomendadas y, en el mejor de los casos, los estudiantes llegarán con dudas e inquietudes relacionadas con la solución de los problemas planteados por el docente. En la investigación, la estrategia del aprendizaje invertido se orientó a fortalecer el potencial autogestivo y colaborativo de los estudiantes al asumir un rol más activo y participativo a través de la toma de decisiones relativas al cómo, cuándo y en dónde aprender, mientras que la presencia del docente se desplazó paulatinamente hacia el 
acompañamiento. Esto implicó centrarse en los intereses y necesidades de los estudiantes e incluir consignas sobre las actividades que el estudiante realizó dentro y fuera de casa, en función de sus estilos y ritmos de aprendizaje.

\section{Fundamentos teórico pedagógicos}

La estrategia didáctica de aprendizaje invertido tiene una orientación socio-constructivista desde las bases teóricas de Vigotsky, que dan una atribución significativa al "medio social y a los tipos de interacciones que realiza el sujeto con los otros" (Rico \& Cobas, 2016, p. 231). Al cambiar la dinámica de la clase con una orientación socio-constructivista, se transforma la dinámica de instrucción centrada en el docente hacia una dinámica del aprendizaje centrada en el estudiante, quien podrá elegir los ambientes y espacios de aprendizaje. Esa posibilidad de elección hace activa la estrategia e incorpora las TIC con un enfoque tecno-pedagógico de carácter recursivo y sistémico para no solamente consultar, sino modificar y adaptar los recursos y actividades formativas desde su casa y desde la escuela. Para facilitar el aprendizaje de manera invertida, el docente adopta un rol de acompañante en la Zona de Desarrollo Próximo (ZDP) denominada así por "la diferencia entre lo alcanzado por la persona de manera individual y lo que es capaz de alcanzar con ayuda de los demás" (Ojalvo, González, Castellanos, Kraftchenko, Molina, Narváez \& Curiel, 2017, p. 57). Es decir, un nivel de desarrollo potencial y un nivel de desarrollo real. En el acompañamiento, el docente utiliza mediadores tales como el lenguaje, a través de consignas para el abordaje de materiales, la búsqueda, consulta y presentación sistemática de la información; también utiliza artefactos digitales y presenciales para la construcción individual de conocimientos en casa, así como la construcción y realimentación colaborativa de conocimientos de mayor complejidad en el aula.

Integrantes de la comunidad digital denominada Red de Aprendizaje Invertido Flipped Learning Network (FLN) ${ }^{1}$ se basan en su experiencia como educadores para definirlo como:

Un enfoque pedagógico en el cual la instrucción directa se moviliza del espacio de grupo de aprendizaje al espacio individual de aprendizaje. La dinámica del aula es transformada en una dinámica de ambiente de aprendizaje interactivo, donde el docente guía a los estudiantes a aplicar conceptos y comprometerse creativamen-

1. Puede consultarse en su sitio web oficial http://flippedlearning.org/ 
te con la asignatura. (Sams, Bergmann, Daniels, Bennett, Marshall \& Arfstrom, 2014 p. 5; traducción propia)

Para esta investigación, el aprendizaje invertido se define como un proceso continuo de reflexión individual y colaborativa derivado de la integración tecno-pedagógica de las TIC y la diversificación de los ambientes de aprendizaje, que se realiza por los estudiantes a partir de actividades autogestivas, con niveles de desarrollo simple, y colaborativas, con niveles de desarrollo complejo; ambas con el acompañamiento del docente.

En el aprendizaje invertido se plantean las bases para transitar hacia otras modalidades educativas, tales como el aprendizaje mixto, semipresencial o blended learning y el aprendizaje móvil o ubicuo.

En la modalidad blended learning el hogar se convierte en otro ambiente de aprendizaje autogestivo y personalizado que se conecta con la escuela a través de la virtualidad. En este aspecto se "combina la instrucción cara a cara con la instrucción mediada por computadora” (Bonk \& Graham, 2012, p. 5).

En la modalidad de aprendizaje ubicuo o móvil, el aprendizaje invertido tiene un impacto al promover el uso de dispositivos móviles así como su uso en la búsqueda y aplicación de la información en contextos de aprendizaje situados en un momento, lugar y circunstancia determinada. De acuerdo con Hwang, Lai y Wang (2015), también ha sido llamado aprendizaje sin problemas, porque conecta espacios y actividades tanto dentro como fuera de la escuela que deben ser diseñadas para motivar a los estudiantes a aplicar lo que han aprendido en ésta en problemas y situaciones de su vida diaria.

Los directores del FLN comunidad de aprendizaje invertido en línea, publicaron cuatro pilares del aprendizaje invertido que los docentes deben incorporar dentro de su práctica (Sams et al., 2014). Estos pilares se relacionan con el contexto de la investigación:

Flexible Environment. Ambiente flexible que se caracteriza por estar sustentado en un conocimiento previo del estudiante, es decir, sus estilos, conocimientos y expectativas respecto a su propio aprendizaje. La flexibilidad también se supedita a las características y necesidades del grupo de aprendizaje.

Learning Culture. La cultura del aprendizaje implica una corresponsabilidad mediada con el estudiante para transitar hacia experiencias de aprendizaje autogestivas y colaborativas. Esta transición se realiza al involucrar a los estudiantes de manera autogestiva en la ejecución de tareas de menor complejidad fuera del aula, mientras que en grupos pequeños, de manera colaborativa dentro de la clase y con la mediación del docente, se abordan las tareas de mayor complejidad. En consecuencia, el estudiante es responsable individual y colaborativamente de sus procesos de aprendizaje. 
Intentional Content. El contenido intencional se precisa en función de seleccionar y elaborar los materiales educativos, objetos de aprendizaje y ambientes que propicien situaciones en las que el estudiante explore tanto individual como colaborativamente los contenidos y adopte estrategias para su propio aprendizaje.

Professional Educator. El educador se profesionaliza en el aprendizaje invertido porque asume una serie de roles que requieren de una formación tecno-pedagógica, flexibilidad en los ambientes de aprendizaje, apertura a desaprender y reaprender de otros, así como la reflexión continua sobre la práctica docente.

Por todo lo anterior, el aprendizaje invertido impacta en los ambientes de aprendizaje, los estilos de aprendizaje, el tiempo, las aplicaciones informáticas, los contenidos y materiales educativos, los roles asumidos y la reflexión. Estos elementos se incluyen como categorías de análisis en la metodología de la investigación proyectiva.

\section{Metodología}

El tipo de investigación realizada en este trabajo es proyectiva de corte cualitativo y transversal. La investigación proyectiva "involucra creación, diseño, elaboración de planes o de proyectos” (Hurtado, 2008, párr. 2). En este caso, la estrategia didáctica está fundamentada en un proceso sistemático en el que los participantes presentan sus necesidades y expectativas a la par de generar procesos explicativos en la propuesta formativa, para así producir los cambios esperados.

La investigación se realizó de septiembre a noviembre de 2015, correspondiente al ciclo escolar 2015-2016. En este periodo se tuvo un número aproximado de 20 sesiones presenciales. Los participantes fueron estudiantes del primer semestre de tres grupos, dos del turno matutino y uno del vespertino. En promedio fueron 30 estudiantes por cada grupo de la unidad de aprendizaje manejo de TIC de la Licenciatura en Ciencias de la Educación.

Las técnicas empleadas, derivadas de la experiencia en el aula, fueron la observación participante y el análisis de contenido de bitácoras reflexivas, publicadas en línea a través de tres grupos cerrados en Facebook.

A partir de la identificación inicial de categorías de análisis realizadas en función de la fundamentación pedagógica del estudio, se realizó un proceso cíclico para la estrategia de aprendizaje invertido que incluyó las fases de planeación, implementación y evaluación (Figura 1). 


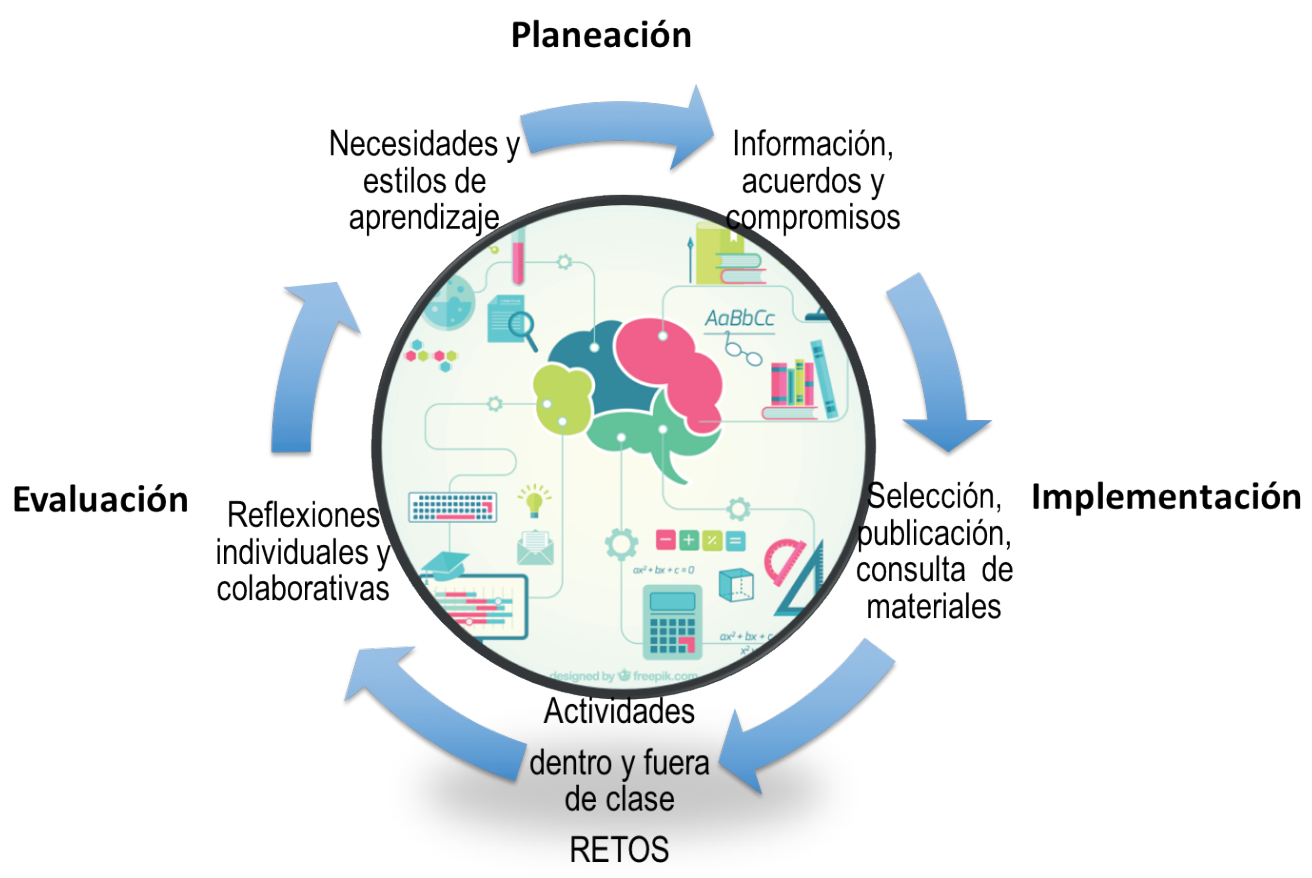

Figura 1. Proceso cíclico de la estrategia de aprendizaje invertido

Fuente: Elaboración propia.

Como parte de la metodología de la investigación proyectiva se describe cada una de las fases abordadas en los grupos de aprendizaje.

\subsection{Planeación de la estrategia}

En esta fase se realizó un diagnóstico previo para conocer los contextos en los que se llevó a cabo la estrategia, así como el diseño de la misma.

Con respecto al diagnóstico, se aplicó un test para que los estudiantes conocieran sus estilos de aprendizaje. El test, aplicado desde la perspectiva de la programación neurolingüística, fue el Visual Auditivo Kinésico (VAK) (SEP, 2014). El objetivo de su aplicación fue promover en los estudiantes el autoconocimiento respecto a sus modos de aprender, por lo que los resultados fueron personales. Una vez realizado lo anterior se les presentó el programa de estudios y la estrategia de clase invertida a través de una infografía (Figura 2). 


\section{¿Qué tengo que hacer?}

1. Reconocerte como un aprendiente invertido que es capaz de aprender dentro y fuera de la escuela, con o sin la ayuda de un docente.

2. Asumir tu responsabilidad y compromiso con tu aprendizaje

3. Buscar e identificar tus pasiones.

4. Trazar tu ruta de aprendizaje

5 Construir tus redes y comunidades de aprendizaje y práctica a través de la Internet $y$ en la presencialidad

6. Determinar tus estrategias para aprender a aprender y hacerlo a lo largo de la vida

\section{¿Por qué el aprendizaje invertido?}

Porque todos somos capaces de aprender, de manera autogestiva y colaborativa, en
contextos más allá del aula de clases y de la escuela

Yo puedo decidir y responsabilizarme de mi

$$
\text { aprendizaje }
$$
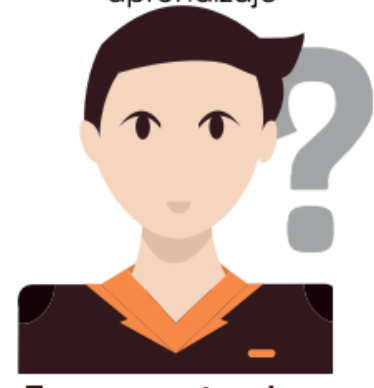

Tres preguntas clave

\section{¿Qué hace el docente y qué hago yo?}

$$
\begin{aligned}
& \text { DOCENTE: } \\
& \text { Me asesora y me acompaña en } \\
& \text { el proceso, si yo lo incluyo. } \\
& \text { YO: Construyo mis redes, } \\
& \text { comunidades y Entorno Personal } \\
& \text { de Aprendizaje } \\
& \text { MIS COMPAÑEROS: Colaboran } \\
& \text { y comparten conmigo en la } \\
& \text { virtualidad y la presencialidad. }
\end{aligned}
$$

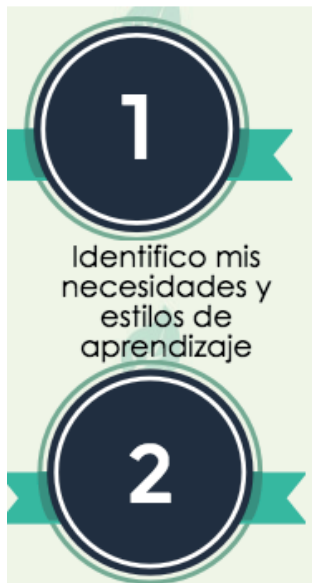

Busco las personas, espacios, artefactos y herramientas web para aprender

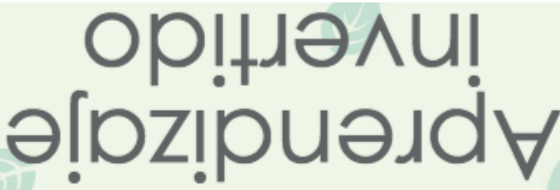

Lo aplico en lo que me gusta

\section{Trazo mi ruta y estrategia de aprendizaje}

Contagio mi entusiasmo y ganas de aprender a otros

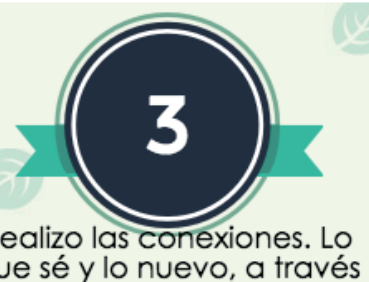
de mis redes y comunidades

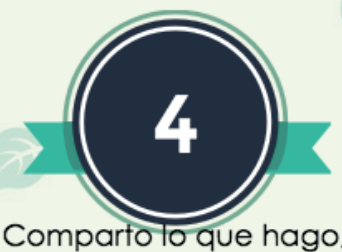

genero contenidos. obtengo realimentación

Figura 2. Infografía sobre el aprendizaje invertido

Fuente: Elaboración propia. 
A la par, se les preguntó a los estudiantes en plenaria presencial lo que necesitaban aprender como parte de los contenidos de la unidad de aprendizaje. Las aportaciones se detallaron en los acuerdos y compromisos grupales e individuales.

Para el diseño, la estrategia se concibe como un proceso didáctico de carácter cíclico centrado en el aprendizaje del estudiante, por lo que se incluyeron aspectos cognitivos, operativos y de intervención. Los aspectos cognitivos se refieren al qué, es decir, a los contenidos que se abordaron con la estrategia a través de los recursos generados o facilitados por la docente-investigadora. Los aspectos operativos se refieren al cómo, o sea, a las actividades que se promovieron como parte de la estrategia; mientras que los aspectos de intervención se enfocan en el para qué, representado por la intención formativa que fue establecida en las competencias a desarrollar por la unidad de aprendizaje.

Además de considerar los aspectos anteriores, para el diseño de la estrategia se sumaron otros elementos, los cuáles se describen a continuación:

Ambientes de aprendizaje. Constituyen una combinación de espacios e infraestructura, y relaciones físicas, sociales, culturales, pedagógicas, psicológicas y didácticas en las que los estudiantes aprenden.

Espacios de aprendizaje. Importantes para generar las demás condiciones del ambiente. Para la aplicación de la estrategia se consideraron los siguientes:

- Espacios virtuales: se consensuó con los estudiantes el uso de tres grupos cerrados en la red social Facebook.

- Espacios presenciales: se trabajó en el salón de clases, el patio y el centro de cómputo de la Escuela.

Consignas. Formaron parte de la comunicación del docente hacia los estudiantes; se caracterizan por expresarse en sugerencias en lugar de indicaciones para el manejo de los recursos y materiales que se incorporan en los espacios virtuales y presenciales. Se priorizó el lenguaje coloquial y de persuasión para llevar a cabo las actividades.

Contenidos. Son los propios del programa de estudios de la unidad de aprendizaje, adaptados a la estrategia invertida. Se consensuó de manera flexible con los estudiantes la oportunidad de que ellos pudieran agregar algunos tópicos de su interés, y de acuerdo con sus necesidades de aplicación, en ésta y otras unidades de aprendizaje.

Evidencias de cumplimiento. Las actividades se transformaron en retos, los cuales se supeditaron a la consulta previa de materiales en casa y a la recuperación de lo aprendido en el espacio del aula. El abordaje de los retos se evidenció en los espacios virtuales y presenciales, a través de la revisión colaborativa de los avances que 
de manera voluntaria proyectan los estudiantes a sus compañeros. Las evidencias de cumplimiento se dieron en dos vertientes: las relacionadas con la consulta previa de los materiales en los espacios virtuales y las que abordaron los retos en los espacios presenciales.

Materiales. El docente los diseñó, creó y adaptó en función de las necesidades de los estudiantes, las características del curso y/o de la temática en particular. Los materiales utilizados se aplicaron en ambientes presenciales y virtuales, éstos fueron:

- Multimedia. Videos, video-tutoriales.

- Gráficos. Infografías.

- Espacios web. Blogs y grupos en redes sociales.

- Cuadernillo digital de retos. Publicado en el sitio Slideshare. ${ }^{2}$

Aprendientes. Todos los estudiantes son responsables y están interesados en su propio aprendizaje. Por ende, son quienes acuerdan la fecha y espacios para su entrega.

Realimentación. La realizó el docente, el equipo de trabajo y los compañeros del grupo. Se orientó a resaltar las oportunidades de mejora más que a la detección y/o corrección de errores, por lo cual se evitaron ciertas palabras tales como: mal, error, reprobado, puesto que pueden desmotivar o ir en detrimento del carácter propositivo para la mejora continua.

En el énfasis de construir colectivamente el conocimiento, Gallardo (2007) establece que "resulta conveniente tratar los siguientes conceptos: a) conocimiento, b) cognición, y c) aprendizaje” (p. 53), los cuales se retoman como parte de la estrategia. Desde esa perspectiva, el conocimiento se define como un proceso de construcción y deconstrucción, propio de la dinámica social que al invertirse replantea una transición de las construcciones autogestivas a colaborativas.

Según Hernández (2001, citado en Gallardo, 2007), "la cognición es el proceso de construcción del conocimiento que involucra las potencialidades del desarrollo y las relaciones que establece el estudiante entre lo que ya conoce y su contexto sociocultural” (p. 54), estas relaciones se fortalecieron con el aula invertida y se evidenciaron en la reflexión individual y grupal, lo que amplió el espectro de lugares y formas de aplicar lo aprendido.

2. El cuadernillo digital de retos se encuentra disponible en: https://es.slideshare.net/ELVIA1308/ manejo-de-tic-problemas-y-ejercicios 


\subsection{Implementación de la estrategia}

De manera presencial, se obtuvo el consentimiento del grupo para su participación dentro de la estrategia. También se explicaron a los estudiantes las intenciones formativas a lograr con la estrategia dentro de la unidad de aprendizaje, redactadas en términos de las siguientes competencias:

1. Desarrolla procesos de autogestión y colaboración atendiendo a sus necesidades de aprendizaje.

2. Aplica las herramientas web en la búsqueda, selección y organización de información en el contexto de su formación académica y profesional.

Previo al trabajo en línea, la docente-investigadora realizó la gestión de los materiales educativos, los cuáles fueron publicados a través de un grupo cerrado en Facebook. La consulta de los materiales permitió a los estudiantes contar con referentes para el abordaje de los retos presenciales (Figura 3).

\begin{tabular}{|c|c|}
\hline $\begin{array}{l}\text { Grupo de trabajo } \\
\text { colaborativo y de } \\
\text { aprendizaje en }\end{array}$ & $\begin{array}{l}\text { Elvia Garduño elimina el incorrecto por favor } \\
\text { Me gusta - Responder - } 25 \text { de septiembre de } 2015 \text { a las } 21: 25 \\
\text { Le guis Corral gracias ya lo hice } \\
\text { Me gusta - Responder - } 01 \cdot 26 \text { de septiembre de } 2015 \text { a las } 14: 46\end{array}$ \\
\hline $\begin{array}{l}\text { Manejo de TIC, } \\
\text { G Grupo cerrado }\end{array}$ & Escribe un comentario... \\
\hline Conversación & Elvia Garduño compartió un enlace. \\
\hline Miembros & 22 de septiembre de 2015 \\
\hline Eventos & $\begin{array}{l}\text { Querid@s aprendientes: } \\
\text { Envío el test de estilos de aprendizaje hay que contestarlo y ANTES DE }\end{array}$ \\
\hline $\begin{array}{l}\text { Fotos } \\
\text { Archivos }\end{array}$ & $\begin{array}{l}\text { ENVIARLO DETERIMINAR CADA QUIEN SU ESTILO CON LA HOJA DE } \\
\text { RESPUESTAS QUE PODRÁN IMPRIMIR. }\end{array}$ \\
\hline & $\begin{array}{l}\text { ESTILOS DE APRENDIZAJE MANEJO DE TIC GRUPO } \\
103\end{array}$ \\
\hline $\begin{array}{l}\text { Buscar en este grupo Q } \\
\text { Accesos directos } \\
\text { (4) MEDI }\end{array}$ & $\begin{array}{l}\text { INSTRUCCIONES: Selecciona únicamente una respuesta para cada pregunta. Para } \\
\text { poder enviar tu cuestionario debe estar completamente contestado. Gracias por tu } \\
\text { participación ANTES DE ENVIAR DETERMINA TU ESTILO UTLIZANDO LA HOJA } \\
\text { DE RESPUESTAS QUE ESTARÁ PUBLICADA EN EL GRUPO DEL FACEBOOK } \\
\text { DOCS.GOOGLE.COM }\end{array}$ \\
\hline Taller de creatividad & It Me gusta Comentar $\quad \rightarrow$ Compartir \\
\hline
\end{tabular}

Figura 3. Captura de pantalla del grupo cerrado de trabajo en la red Facebook.

Fuente: https://www.facebook.com/groups/1477749252527944/ 
Como parte de la implementación de la estrategia, los estudiantes realizaron las siguientes actividades:

3. La exploración de materiales digitales cortos en espacios virtuales dirigidos por la docente-investigadora, pero paulatinamente controlados por los estudiantes, publicados en grupos en Facebook o entregados directamente para su consulta fuera de la clase.

4. La contestación de cuestionarios de cinco preguntas abiertas, sobre los materiales publicados para la consulta en casa.

5. La contestación de preguntas indagadoras al inicio de la clase para recuperar lo aprendido en los materiales y espacios de consulta e iniciar los retos presenciales.

6. La aplicación de lo aprendido en retos flexibles de mayor complejidad cognitiva para realizarse en el tiempo de clase. Estos retos tuvieron un abordaje contextualizado y se realizaron individualmente o en equipo.

7. La realimentación grupal y de la docente-investigadora en clase para compartir los avances en los retos asumidos.

8. La publicación y difusión de las evidencias obtenidas como solución al abordaje de los retos asumidos.

9. La elaboración de bitácoras compartidas en espacios virtuales, así como preguntas de cierre en la sesión presencial para la reflexión metacognitiva.

La gestión de las actividades anteriores ha generado diferentes evidencias representadas por cuestionarios de diagnóstico de necesidades, expectativas y propuestas de aprendizaje: un test sobre estilos de aprendizaje; infografías sobre el aprendizaje invertido y temáticas relacionadas; Objetos Digitales de Aprendizaje (ODA) creados y adaptados; un cuadernillo de retos digitales; una propuesta de secuencia didáctica para el trabajo en aula invertida, y una conferencia taller sobre planeación didáctica invertida presentada en la Escuela Superior. Actualmente se imparte un Massive Open On Line Course o Curso Masivo Abierto en Línea (MOос) sobre el aprendizaje invertido en la comunidad digital de aprendizaje Canvas. ${ }^{3}$

3. El MOoc sobre aprendizaje invertido estuvo disponible hasta el 6 de agosto de 2017 en https:// www.canvas.net/browse/uag-es/courses/aprendizaje-invertido 
Elvia Garduño Teliz, Colette Marie Dugua Chatagner

\subsection{Evaluación de la estrategia}

En esta fase se presentaron evidencias de aprendizaje y del proceso de investigación de la propia estrategia.

Las evidencias de aprendizaje son en su mayoría digitales y se encuentran elaboradas en aplicaciones informáticas. Al respecto, la evaluación consistió en calificar los retos conforme a los criterios establecidos en listas de cotejo. A la par se realizó la realimentación tanto preliminar como final de manera presencial, en el aula de clases y en el centro de cómputo, por los estudiantes y de manera virtual por la docenteinvestigadora.

Destacó la autoevaluación reflexiva de los estudiantes en todo el proceso al contestar individual y colaborativamente las bitácoras, así como las actividades de coevaluación cualitativa donde se centraron básicamente en identificar oportunidades y sugerir acciones de mejora. La heteroevaluación se sustentó en listas de cotejo previamente dadas a conocer a los estudiantes. En las sesiones presenciales, la construcción de bitácoras colaborativas motivó a los estudiantes a participar de manera activa en la realimentación de sus propios compañeros y fortaleció la comunicación grupal para la generación de ideas, cuestionamientos y sugerencias a los trabajos presentados por sus pares.

En cuanto a la calificación, las ponderaciones se consensuaron previamente con el grupo y se decidió incorporar puntos extra como mecanismo para favorecer la actitud hacia el aprendizaje y disminuir la preocupación por la calificación.

Las evidencias del proceso de investigación realizadas por los estudiantes son principalmente reflexivas y se encuentran contenidas en las bitácoras individuales publicadas en los grupos de Facebook. De estas evidencias, se realizó el análisis de contenido conforme a las categorías que fueron establecidas previamente.

\section{Análisis de categorías}

El análisis de las categorías indicó cambios, adaptaciones y mejoras en la estrategia. Esto implicó un proceso paulatino para los estudiantes, puesto que manifestaron la necesidad de intervención directa del docente para dictar lo que debe aprenderse, cómo debe aprenderse y lograr las competencias esperadas. A continuación se presenta el análisis de las categorías derivado de la conjunción de la observación y el análisis de contenido: 
Ambientes de aprendizaje. Se estimó $80 \%$ de contenidos presenciales y $20 \%$ de contenidos en línea, por lo cual se trabajó en una modalidad blended learning.

La inclusión del blended learning favoreció la exploración de otros espacios de aprendizaje tanto virtuales como presenciales. Se valoró positivamente por los estudiantes la flexibilidad en la elección de los lugares presenciales para trabajar (aula, centro de cómputo, patio de la escuela), así como la inclusión de grupos cerrados en Facebook que usaron posteriormente para otras unidades de aprendizaje.

Estilos de aprendizaje. Al conocer los resultados de su test los estudiantes eligieron qué estilos querían movilizar mayormente, prevaleciendo el visual y el quinésico en los contenidos y actividades de aprendizaje. No obstante, al finalizar la aplicación de la estrategia y de manera personalizada se les dieron orientaciones para trabajarlos. En estas orientaciones destacó el estilo auditivo como el de menor puntaje en el test.

Tiempo. Para la consulta y actividades de aprendizaje fuera de clase se estimó un tiempo de entre una y dos horas. En las actividades de clase, el tiempo se limitó a dos horas. No obstante, fue insuficiente para completar la revisión y realimentación a todos los equipos de trabajo. Con algunos grupos, se agregó una sesión para concretar los retos. Los propios estudiantes establecieron las fechas en las que se comprometían grupalmente a entregar las evidencias de aprendizaje.

La flexibilidad en el tiempo complicó el establecimiento de los periodos de evaluación para la docente-investigadora, pero fue vista de manera favorable por los estudiantes, puesto que ellos tuvieron injerencia y decisión sobre su manejo.

Pese a lo anterior, no hubo problemas para reportar en tiempo y forma las evaluaciones de los tres grupos.

Aplicaciones informáticas. La elección de la aplicación informática a utilizar estuvo determinada por el nivel de manejo y de conocimiento de herramientas por parte de los estudiantes.

Los niveles de manejo fueron diferentes, desde principiantes hasta autónomos. Para trabajar con los principiantes se plantearon asesorías extra a las cuales asistieron. No obstante, los estudiantes prefirieron la asesoría de sus pares, al agruparse en equipos pequeños de trabajo fuera de clase, y el apoyo de la docente-investigadora dentro de ésta.

El nivel de conocimiento de las aplicaciones informáticas se orientó más hacia la ofimática: procesadores de textos, presentadores de diapositivas. Existen necesidades de aprendizaje en el conocimiento de herramientas web para ampliar los niveles de uso más que los de manejo. 
Contenidos y material educativo. Para aplicar la estrategia, se realizó una adaptación al programa de estudios de la unidad de aprendizaje. Esta adaptación ofreció la oportunidad de incluir contenidos y materiales por parte de los estudiantes. Es decir ellos señalaron lo que querían aprender. En los tres grupos se dieron diferentes temáticas como la edición de videos, la gestión de archivos en Internet, la inserción de elementos formales en la estructura de documentos en Word, por mencionar algunos de ellos. Los estudiantes subieron video-tutoriales y compartieron archivos para mejorar la presentación de las prácticas y el abordaje de los retos.

Rol del docente. Para el acompañamiento se asumieron en todos los grupos los roles de experto en la disciplina y mediador, facilitador y asesor. La ejecución de roles estuvo supeditada a los niveles de uso y manejo presentados por los estudiantes. En este sentido, no hubo oportunidades de asumir el rol de tutor y de co-aprendiente.

Rol del estudiante. Si bien el trabajo en equipo se hizo de manera empática y a voluntad de los estudiantes, hay una tendencia hacia el aprendizaje individual, aislado y receptivo.

Reflexión. Este proceso se hizo tanto de manera presencial a través de la interacción grupal, como de manera individual en las sesiones de casa. En ambos sentidos se hicieron preguntas derivadas de las bitácoras como, por ejemplo: ¿Qué hice? ¿Qué sentí? y ¿Qué aprendí? La reflexión colaborativa realizada de manera presencial en plenaria fue más amplia que la virtual. Estuvo supeditada a los comentarios de ciere de sesión realizados de manera presencial, así como a la bitácora invertida que se refiere a lo que ha realizado el alumno fuera de clase en apoyo a su aprendizaje. La limitante principal para profundizar en este análisis fue el tiempo efectivo de clase para compartirlo entre el grupo, así como la forma sucinta en la que los estudiantes respondieron a las preguntas de la bitácora para la Comprensión Ordenada del Lenguaje (COL).

El análisis de lo observado, así como del contenido establecido en las bitácoras, determinó la funcionalidad de la estrategia, es decir, su facilidad de uso tanto en el logro de los aprendizajes como en la intención formativa; esto se concentra en la Tabla 1. 
Tabla 1. Funcionalidad de la estrategia

\begin{tabular}{ll}
\hline \multicolumn{1}{c}{ Unidades de análisis } & \multicolumn{1}{c}{ Funcionalidad } \\
\hline Ambientes de aprendizaje & Integra ambientes mixtos formales e informales. \\
\hline Estilos de aprendizaje & $\begin{array}{l}\text { Combina elementos visuales, auditivos y quinésicos en los } \\
\text { contenidos y actividades de aprendizaje. }\end{array}$ \\
\hline Tiempo & Genera flexibilidad en el manejo del tiempo de clase. \\
\hline Aplicaciones informáticas & $\begin{array}{l}\text { Promueve el conocimiento y manejo de diversas aplicaciones } \\
\text { y herramientas. }\end{array}$ \\
\hline Contenidos y material educativo & Favorece la creación y curación de contenidos. \\
\hline Rol del docente & $\begin{array}{l}\text { Diversifica sus acciones basadas en el acompañamiento hacia } \\
\text { el aprendizaje. }\end{array}$ \\
\hline Rol del estudiante & $\begin{array}{l}\text { Toma decisiones y acciones basadas en su compromiso y } \\
\text { responsabilidad con su aprendizaje. }\end{array}$ \\
\hline Reflexión & Promueve procesos metacognitivos individuales y colectivos. \\
\hline
\end{tabular}

Fuente: Elaboración propia.

La creación de ambientes mixtos con la estrategia de aprendizaje invertido se inscribe como una innovación para el desarrollo de competencias, al reforzar el proceso educativo centrado en el estudiante, el aprendizaje significativo, el cambio en los roles y la evaluación del aprendizaje. En este sentido, la estrategia de aprendizaje invertido es un proceso ligado a la realimentación, la reflexión y la metacognición.

\section{Resultados y discusión}

$\mathrm{Al}$ ser una experiencia completamente vivencial en la práctica educativa, los resultados se presentan en función de las reflexiones realizadas mediante preguntas generadoras en las sesiones presenciales, así como las bitácoras invertidas COL, denominadas así puesto que son reflexiones originadas por la autogestión de las actividades realizadas fuera de clase.

Los resultados se agruparon en unidades de análisis referidas por los estudiantes en sus reflexiones:

Los aprendizajes obtenidos. Los estudiantes identificaron tanto los retos como los recursos, contenidos y actividades utilizadas para su solución, presentación y pu- 
blicación. En su redacción denotaron niveles de conocimiento desde la comprensión y la explicación hasta la aplicación. En este último estrato hay niveles de dominio de las TIC relacionados con la implementación de la estrategia, sus aprendizajes adquiridos así como sus conocimientos previos, principalmente en el manejo del software. No obstante, todos señalaron que es la primera vez que trabajan con esta estrategia y aplican las TIC para el abordaje de retos, que pueden adaptar eligiendo variantes seleccionadas.

Lo que más les ha gustado. Todos los estudiantes, sin excepción, mostraron agrado hacia la dinámica de la clase invertida; expresaron su interés en la consulta de los recursos, ya que les pareció atractivo por su corta duración y que el trabajo más complejo se realizó en clase. Algunos estudiantes manifestaron dificultades en el manejo de herramientas como Facebook y la descarga de los recursos, pero fueron solucionadas con el apoyo de sus compañeros. La libertad en la elección de las fechas de entrega de trabajo, lo que desean aprender complementariamente a lo establecido en el programa, la flexibilidad en el abordaje de los retos y la toma de decisiones grupales, son aspectos calificados como positivos. Como parte de la dinámica del aprendizaje invertido, la realización de las bitácoras y de las preguntas reflexivas de cierre les es poco familiar y afirman no haberlo trabajado con ningún otro docente.

Lo que menos les ha gustado. Los grupos en general mostraron descontento por el número limitado de equipos de cómputo disponibles en la institución, además de la conectividad a Internet, que es muy limitada y no acorde con los requerimientos de una Licenciatura. También expresaron su inconformidad respecto a las instalaciones del centro de cómputo, ya que existe poco espacio para atender a cada grupo. Las inconformidades fueron en cuestiones de infraestructura.

Sugerencias de mejora para la clase. Las sugerencias estuvieron relacionadas principalmente con las inconformidades del grupo respecto a la infraestructura. También se hicieron respecto a una mayor participación y motivación en las sesiones presenciales para facilitar el aprendizaje colaborativo. Algunos estudiantes pidieron una realimentación más personalizada, otros pidieron mayor presión en los tiempos de clase, ya que no se trabajó de manera homogénea en cuestiones de entrega de trabajos. Finalmente, hicieron sugerencias relacionadas con una mayor mediación en línea para la comprensión de los contenidos fuera de clase.

Niveles de logro. Considerando que el desarrollo de competencias es un proceso continuo cuya movilización y transferencia se realiza en diferentes situaciones, contextos y momentos, los estudiantes manifestaron la inducción hacia procesos auto- 
gestivos y colaborativos relacionados con sus necesidades de aprendizaje. Respecto a la aplicación de herramientas web, lo observado en el proceso denota una necesidad manifiesta para atender la gestión de información, para lo cual se requerirá un proceso de formación específica. En ese sentido, la alfabetización informacional debiera darse como parte del currículo, además de generar las condiciones para aplicarse como parte de la estrategia de aprendizaje invertido.

Por todo lo anterior, la aplicación de la estrategia demostró ser de utilidad para facilitar el aprendizaje invertido, asumir diferentes roles, cambiar la dinámica del grupo y facilitar el logro de las competencias establecidas en la unidad de aprendizaje.

La dinámica de aplicación de la estrategia en los tres grupos de aprendizaje fue distinta. El tiempo disponible en la presencialidad fue una limitante para atender completamente a las necesidades grupales, debido al número de sesiones, duración y suspensiones oficiales de clases. No obstante, esto se logró subsanar a través de los medios virtuales y asesorías extra clase, solicitadas por los estudiantes.

En cada grupo existió un número aproximado de tres a cinco estudiantes que mostraron cierta resistencia a realizar algunas tareas, por ejemplo, los cuestionarios cortos que requieren la consulta y comprensión de los materiales. Esto implicó una mayor mediación y compromiso personal por parte del docente-facilitador, así como la prórroga de los tiempos de entrega, situación que no demeritó la credibilidad y compromiso del grupo con la unidad de aprendizaje.

$\mathrm{Al}$ incluir el aprendizaje invertido en la planeación, ejecución y evaluación de su curso, tanto el estudiante como el docente asumen diversos roles, en los que movilizan sus competencias digitales y tecno-pedagógicas, para aprovechar las TiC en los procesos formativos.

Algunas investigaciones sobre el aprendizaje invertido señalan que "al exponer por primera vez a los estudiantes a un modelo invertido, se muestran renuentes, aceptando dicha estructura al avanzar el curso y después de percibir ganancias" (Baepler et al., 2014; Mason et al., 2013; Tune et al., 2013)" (Martínez, Esquivel \& Martínez, 2014 , pp. 154). Sin embargo, en la investigación los estudiantes manifestaron disponibilidad, a pesar de subsistir cierto condicionamiento hacia un aprendizaje receptivo y ciertas dificultades en el uso y manejo de las TiC.

En otra investigación análoga de corte cuasi experimental, la percepción de los estudiantes que estudian la Licenciatura en educación primaria sobre una estrategia de enseñanza-aprendizaje universitaria con un enfoque invertido, señala una "preferencia por las metodologías activas y dinámicas” (Martín \& Tourón, 2017, p. 208); 
además de una mejora significativa en las competencias establecidas como dimensiones del estudio. Sin embargo, en las investigaciones las percepciones favorables sobre el aprendizaje invertido no suponen la alfabetización digital. Al igual que en esta investigación, se requiere mejorar los procesos de alfabetización informacional y digital por parte de los estudiantes y profesores, lo cual puede originar nuevas líneas de investigación que incidan en la gestión curricular, el aprendizaje y la práctica docente.

En consecuencia, dentro de las aplicaciones prácticas del trabajo se tienen en perspectiva algunas áreas de oportunidad que impactan la mejora continua de la estrategia, tales como: la incorporación del aprendizaje invertido en los programas de estudio y programas de acompañamiento, la integración de la gestión de los Objetos Digitales de Aprendizaje para docentes y estudiantes, así como la aplicación de la modalidad mixta o blended learning en los ambientes de aprendizaje de la Licenciatura.

\section{Conclusiones}

El mundo virtual nos ha alcanzado y estamos en presencia de transformaciones radicales en pedagogía, con la existencia de espacios virtuales como ambientes de aprendizaje que facilitan interacciones sociales entre los participantes de los procesos educativos. Se trata entonces de construir un modelo pedagógico basado en la cultura de aprender y ya no de enseñar, con un estudiante activo, agente de su propia formación, sobre la base de la pedagogía constructivista y el uso sistemático de las Tic en una interacción permanente facilitador-estudiante, tanto individual como grupal (Eusse, 2006, pp. 237-240).

La estrategia del aprendizaje invertido transformó la dinámica de la clase y del aprendizaje, al tomar como punto de partida las necesidades y expectativas de los estudiantes. Los resultados en la aplicación de esta estrategia denotaron actitudes favorables respecto a invertir la clase, los aprendizajes y los roles. No obstante, el reto mayor es contrarrestar muchos de los aspectos tradicionales que han marcado al docente como transmisor y al estudiante como receptor; por ende, los resultados obtenidos en este trabajo muestran una clara iniciativa y compromiso de los estudiantes con su aprendizaje, situación que en el futuro de la Licenciatura en Ciencias de la Educación, es de trascendencia formativa. 


\section{Lista de referencias}

Bishop, J. L., \& Verleger, M. A. (Junio, 2013). The flipped classroom: A survey of the research. Trabajo presentado en la National Conference Proceedings de la American Society for Engineering Education, Atlanta, Georgia. Recuperado de http://www.asee.org/public/conferences/20/papers/6219/view

Bonk, C., \& Graham, C. (2012). The handbook of blended learning: Global perspectives, local designs. Estados Unidos: John Wiley \& Sons.

Díaz-Barriga, F., \& Hernández, G. (2002). Estrategias docentes para un aprendizaje significativo. Una interpretación constructivista ( $2^{\mathrm{a}}$ ed.). México: McGraw Hill.

Eusse, O. (2006). De la docencia presencial a la asesoría a distancia: tránsito necesario. En C. Barón (Coord.), Proyectos educativos innovadores. Construcción y debate (pp. 237-240). México: Centro de Estudios sobre la Universidad-Universidad Nacional Autónoma de México.

Flores, G., Montaño, A., Herrera, O., Endoqui, K., \& García, G. (2017). El lenguaje, una herramienta para aprender mediante el modelo de gestión Aprendizaje Invertido, un estudio de caso en alumnos de $5^{\circ}$ de primaria. Edutec-e. Revista Electrónica de Tecnología Educativa, 59, 1-17. Recuperado de http://www.edutec.es/revista/index.php/edutec-e/article/view/756

Gallardo, S. C. (2007). El constructivismo social como apoyo al aprendizaje en línea. Revista Electrónica Nueva Época, 7(7), 46-62. Recuperado de http://www.udgvirtual.udg.mx/apertura/index.php/apertura/article/view/1209

Hurtado, J.(2008). La investigación proyectiva [Mensaje en un Blog]. Recuperado de http:// investigacionholistica.blogspot.mx/2008/02/la-investigacin-proyectiva.html\#

Hwang, G.-J., Lai, C.-L., \& Wang, S.-Y. (2015). Seamless flipped learning: a mobile technology-enhanced flipped classroom with effective learning strategies. Journal of Computers in Education, 2(4), 449-473. doi:http://doi.org/10.1007/ S40692-015-0043-0

Martín, D., \& Tourón, J. (2017). El enfoque flipped learning en estudios de magisterio: percepción de los alumnos. RIED. Revista Iberoamericana de Educación a Distancia, 2o(2) 187-211. Recuperado de http://revistas.uned.es/index.php/ ried/article/view/17704

Martínez, W., Esquivel, I., \& Martínez, J. (2014). Aula invertida o Modelo invertido de aprendizaje: Origen, sustento e implicaciones. En I. Esquivel (Coord.), Los Modelos Tecno-Educativos, Revolucionando El Aprendizaje Del Siglo XxI (pp. 
143-160). Recuperado de http://eduteka.icesi.edu.co/pdfdir/modelos-tecnoeducativos.pdf

Nederveld, A., \& Berge, Z. L. (2015). Flipped learning in the workplace. Journal of Workplace Learning, 27(2), 162-172.

Ojalvo, V., González, M., Castellanos, A., Kraftchenko, O., Molina, A., Narváez, A., \& Curiel, L. (2017). Comunicación educativa una invitación al diálogo. La Habana, Cuba: Editorial Universitaria Félix Varela.

Rico, P., \& Cobas, C. (2016). Aprendizaje y desarrollo. En B. Capote (Ed.), Fundamentos de Psicología para escuelas pedagógicas (pp. 228-274). La Habana, Cuba: Pueblo y educación.

Sales, D., \& Pinto, M. (Eds.). (2016). Pathways Into Information Literacy and Communities of Practice: Teaching Approaches and Case Studies. Reino Unido: Chandos Publishing.

Sams, A., Bergmann, J., Daniels, K., Bennett, B., Marshall, H., \& Arfstrom, K. (2014). The four pillars of F-L-I-P. Flipped Learning Network (FLN). Recuperado de http://flippedlearning.org/wp-content/uploads/2016/o7/FLIP handout FNL Web.pdf

Santos, B. (2016). Aprendizaje invertido y estilos de aprendizaje: el caso de estudiantes universitarios en Administración. Universidad Interamericana para el Desarrollo. Ponencia presentada en Virtual Educa, Puerto Rico. Recuperado de http:// acceso.virtualeduca.red/documentos/ponencias/puerto-rico/1289-db2b.pdf

Secretaría de Educación Pública. (2014). Manual de Estilos de Aprendizaje. Material autoinstruccional para orientadores educativos. Recuperado de http://biblioteca.ucv. cl/site/colecciones/manuales u/Manual Estilos de Aprendizaje 2004.pdf

Sierra, R. (2008). La estrategia pedagógica, su diseño e implementación. La Habana, Cuba: Pueblo y educación.

Talbert, R. (2014). Toward a common definition of "flipped learning" [Mensaje en un Blog]. Recuperado de http://www.chronicle.com/blognetwork/castingoutnines/2014/04/o1/toward-a-common-definition-of-flipped-learning/

Vidal, M., Rivera, N., Nolla, N., Morales, I., \& Vialart, M. (2016). Aula invertida, nueva estrategia didáctica. Educación Médica Superior, 3o(3), 678-688.

Yarbro, J., Arfstrom, K., McKnight, K., \& McKnight, P. (2014). Extension of a review of flipped learning. Estados Unidos: George Mason University/Pearson. Recuperado de http://flippedlearning.org/wp-content/uploads/2016/o7/Extensionof-FLipped-Learning-LIt-Review-June-2014.pdf 